\title{
The Varioscope AR - A Head-Mounted Operating Microscope for Augmented Reality
}

\author{
Wolfgang Birkfellner ${ }^{1}$, Michael Figl ${ }^{1}$, Klaus Huber ${ }^{1}$, Franz Watzinger ${ }^{2}$, \\ Felix Wanschitz ${ }^{2}$, Rudolf Hanel $^{3}$, Arne Wagner ${ }^{2}$, Dietmar Rafolt ${ }^{1}$, Rolf Ewers ${ }^{2}$, \\ and Helmar Bergmann ${ }^{1,4}$ \\ 1 Department of Biomedical Engineering and Physics \\ Wolfgang.Birkfellner@univie.ac .at \\ 2 Department of Oral and Maxillofacial Surgery \\ 3 Department of Diagnostic Radiology, Division of Osteoradiology \\ ${ }^{4}$ Ludwig-Boltzmann Institute of Nuclear Medicine \\ All: General Hospital, University of Vienna, \\ Waehringer Guertel 18-20, A-1090 Vienna, Austria
}

\begin{abstract}
Inherent to the field of computer-aided surgery (CAS) is the necessity to handle sophisticated equipment in the operating room; an undesired side-effect of this development is the fact that the surgeon's attention is drawn from the operating field since surgical progress is partially monitored on the computer's screen. The overlay of computergenerated graphics over a real-world scene, also referred to as augmented reality (AR), provides a possibility to solve this problem. The considerable technical problems associated with this approach such as viewing of the scenery within a common focal range on the head-mounted display (HMD) or latency in display on the HMD have, however, kept AR from widespread usage in CAS. In this paper, the concept of the Varioscope AR, a lightweight head-mounted operating microscope used as a HMD, is introduced. The registration of the patient to the preoperative image data as well as preoperative planning take place on VISIT, a surgical navigation system developed at our hospital. Tracking of the HMD and stereoscopic visualisation take place on a separate POSIX.4 compliant realtime operating system running on PC hardware. While being in a very early stage of laboratory testing, we were able to overcome the technical problems described above; our work resulted in an AR visualisation system with an update rate of $6 \mathrm{~Hz}$ and a latency below 130 ms. First tests with 2D/3D registration have shown a match between $3 \mathrm{D}$ world coordinates and 2D HMD display coordinates in the range of 1.7 pixels. It integrates seamlessly into a surgical navigation system and provides a common focus for both virtual and real world objects. On the basis of our current results, we conclude that the Varioscope AR with the realtime visualisation unit is a major step towards the introduction of AR into clinical routine.
\end{abstract}




\section{Introduction}

Augmented reality (AR), the overlay of computer-generated graphics over a real world scene, has a tantalising potential for visualisation in computer-aided surgery (CAS). Several groups have tried to achieve this goal. One of the earliest approaches was the integration of the computer's monitor into the operating microscope for neuronavigation $8[10,11,13,16]$. While this appears to be the most promising technology for clinical applications where an operating microscope is used (such as in neuro- or skull base surgery), the vast majority of surgical specialties does not utilise such a device, and the introduction of such an expensive system for the purpose of AR-visualisation alone appears problematic. Others have tried to use commercial head-mounted displays (HMD) 9,18, or semi-transparent panels [7 for displaying monitor information. The major problem of this approach is the fact that a common focus of the real world scene and the computer-generated graphics cannot be achieved by the viewer's eye. A possibility to overcome this problem is the usage of miniature video cameras $[12$ and monitors [9]. The image from the video cameras can be merged with the computer-generated scene and displayed on the video monitors. The obvious drawback of this approach is, however, the fact that in addition to the HMD the surgeon also has to wear the miniature video cameras on the headset, and that a video generated view cannot compete with the view of a scenery as provided by an optical system alone in terms of image quality.

From our clinical experience, a number of requirements was defined for AR visualisation in CAS:

- Surgical instruments such as operating microscopes are preferrable for AR visualisation since clinical acceptance is easier to be achieved.

- Common focus for both the real-world scene and the computer-generated graphics has to be provided.

- Display latency due to lags in position measurement and rendering time requirements have to be minimised to avoid simulator sickness 2 .

- The AR visualisation has to be an add-on to a normal navigation system. Sophisticated image processing such as multiplanar reformatting and volume rendering still have to be available during surgery while not overloading the scenery displayed in the HMD.

- Economic and intraoperative time expenses due to AR have to be kept to a reasonable amount.

These considerations led to the development of the Varioscope AR, a miniature head mounted operating microscope for surgical navigation; it features display of additional computer generated sceneries and communication to a surgical navigation system. 


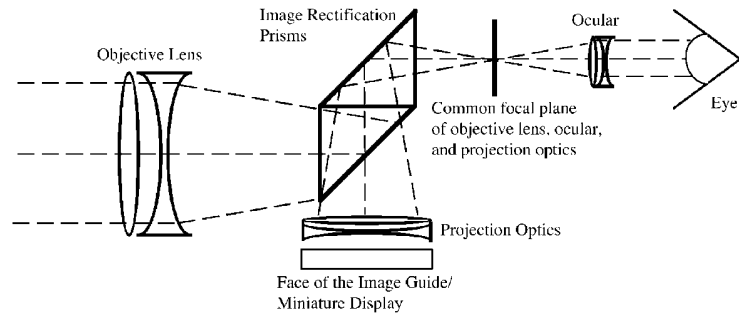

\begin{tabular}{c|c}
\hline Working distance & $300-600 \mathrm{~mm}$ \\
\hline Magnification & $3.6 \times-7.2 \times$ \\
\hline Parallax correction & Automatic \\
\hline Weight & 297 grams \\
\hline Physical dimensions & $120 \mathrm{~mm}$ width, $73 \mathrm{~mm}$ length \\
\hline
\end{tabular}

Fig. 1. The principle of image overlay in the Varioscope AR. An additional image from a miniature computer display is being projected into the focal plane of the Varioscope's objective lens. Both images can be viewed through the ocular of the Varioscope.

Table 1. Optical data of the Varioscope.

\section{Materials and Methods}

\subsection{Visualisation Optics}

The Varioscope is a head-mounted operating microscope developed and marketed by Life Optics, Vienna/Austria (http://www.lifeoptics.com). A list of the Varioscope's optical data can be found in Table 1 A beamsplitter together with a projection lens was inserted into the optical path by Docter Optics, Vienna/Austria. Both the image from the Varioscope's objective lens and the projection optics merge in the focal plane of the objective lens. Thus both the real world scene and the computer graphics can be viewed through the Varioscope's ocular and appear focused to the the viewer's eye (Fig. 11).

\subsection{Display System}

Two miniature VGA displays (AMEL HiBrite, Planar Systems, Munich/Germany, http:/(www.planar.com) with $640^{*} 480$ pixel resolution and 0.75 inch display diameter were connected to a miniaturisation lens system which reduces the image from the display by a factor of 0.67 . This image is being transferred to the projection optics of the Varioscope AR by means of a flexible, high resolution image guide with a resolution of $800^{*} 1000$ pixels and an active area of $8^{*} 10 \mathrm{~mm}$ (Schott Fiber Optics, Southbridge/MA, http://www.schottfiberoptics.com).

\subsection{HMD Tracking and Calibration}

The HMD is being tracked by an optical tracking system (Flashpoint 5000, Image Guided Technologies, Boulder/CO, http://www.imageguided.com). A LED assembly was mounted to the Varioscope AR; a triaxial gyroscope (ATA ARS09) and three accelerometers (Endevco $7290 \mathrm{~A}$ ) are rigidly connected to the LED 


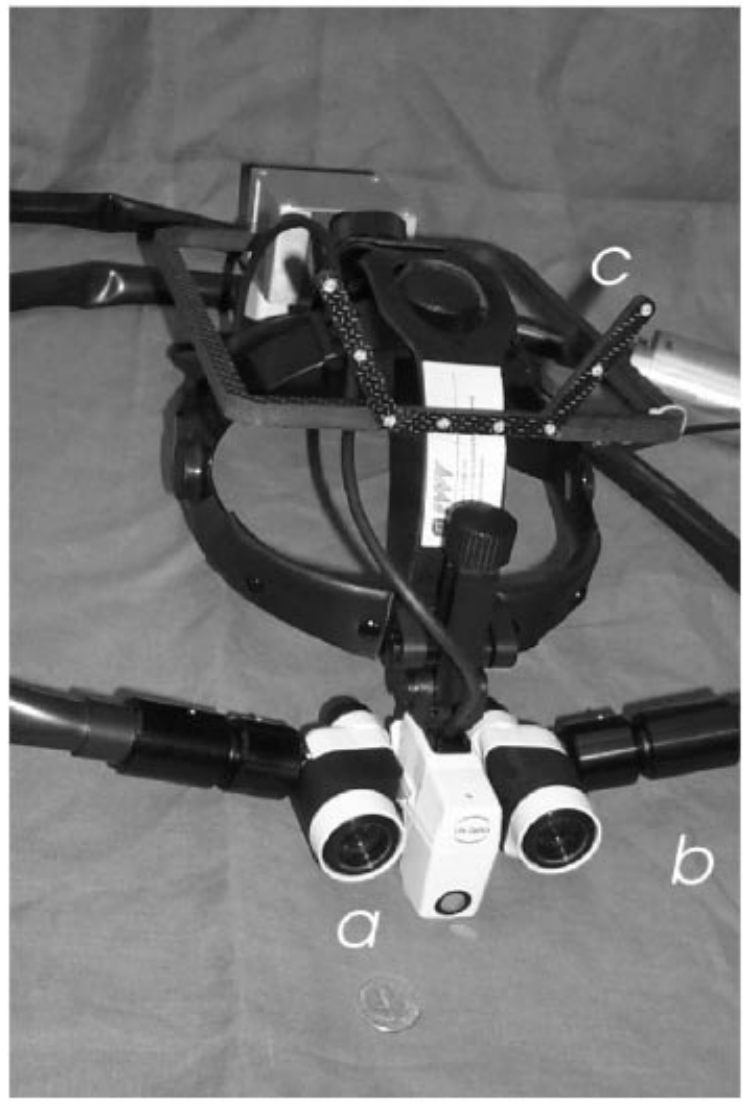

Fig. 2. The first prototype of the Varioscope AR. In addition to the normal Varioscope (a), two image guides (b) injecting a scene generated by the realtime control unit of the Varioscope are attached. Furthermore, a LED assembly (c) rigidly connected to a triaxial gyroscope and accelerometer assembly can be seen.

assembly (Fig. 21). The readings from the kinematic sensors are to be used for predictive filtering of the HMD's position through a Kalman filter 114. At the very moment, the HMD is, however, only tracked by the optical tracker.

Photogrammetric registration of the readings from the optical tracker to the actual scene to be viewed is achieved by Tsai's algorithm [17. While this is work in progress, we have achieved first results by using a variant of Tsai's algorithm which uses a coplanar 3D world coordinate data set. These data were retrieved by aiming a crosshair displayed in th HMD at a calibration grid. From these data, six extrinsic calibration parameters (a rotation and a translation which transfers the world coordinates to the HMD's coordinate system) and two intrinsic parameters (the effective focal length of the HMD's optics and the radial distortion coeficient) are determined. Three more parameters (the center of the display relative to the optical axis and an uncertainty factor which is of no interest for this application) were either determined manually or omitted. 


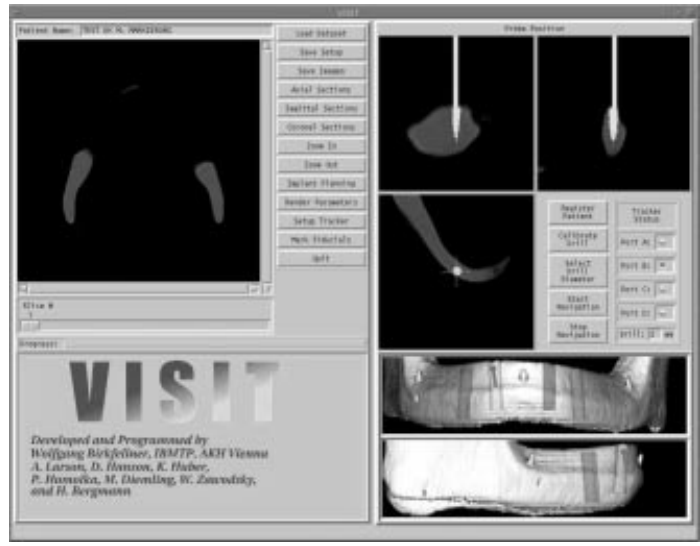

Fig. 3. A screenshot of VISIT, the non-realtime navigation system for additional visualisation on multiplanar reformatted slices and volume renderings. VISIT acquires data from the RT-control system for tool position visualisation, and delivers the data for generating the OpenGL model in the coordinate frame of the patient to the RTcontrol system. This OpenGL model is then visualised on the Varioscope AR (see Fig. 4).

\subsection{HMD Control and Communication with a Navigation System}

The visualisation of the preoperative scene takes place on a POSIX.4 compliant real-time operating system (Lynx OS 3.0.1, http://www.lynx.com) running on a standard PC (Intel Pentium II Processor, $450 \mathrm{MHz}, 128 \mathrm{MB}$ RAM) with a standard Ethernet controller (3com 509B), a SCSI controller (Adaptec 2940UW), two graphics controllers (Matrox Millenium II), and an 8 channel ADC board with $200 \mathrm{kHz}$ sampling rate (Pentland Systems LM1, http://www.pentland.co.uk). Two independent $\mathrm{X}$-servers are driven by the Lynx OS. OpenGL programming was done using the Mesa 3.0 API (http://www.mesa3d.org) and the GLUTtoolkit [15].

The realtime system polls data from the optical tracker at an update rate of approximately $10 \mathrm{~Hz}$. These data are used to render two perspective scenes on the VGA displays according to the actual position of the HMD, the patient and the surgical tool (Fig. (4). Furthermore, a navigation system is connected to the real-time system. VISIT, the system used for these experiments, was developed at our hospital (Fig. 3); it's first application is the computer aided insertion of endosteal implants in the field of cranio- and maxillofacial surgery [3/4,5]6]. The navigation system acquires data at a lower priority (approx. $1 \mathrm{~Hz}$ ). It visualises the drill's position on obliquely reformatted slices and volume renderings. The accuracy of the system was found to be $0.9 \pm 0.4 \mathrm{~mm}$ [4. The preoperative planning data are sent to the realtime system after patient-to-image registration; the real-time system derives the OpenGL scene from these data (Fig. 51). Communication between the CAS-workstation and the realtime system takes place by means of POSIX.1 conformant non-canonical serial communication via the RS232 interface. The system waits for $0.1 \mathrm{~s}$ for a request from the CAS system; if the CAS-system does not send a request, the next position dataset is polled from the optical tracker, and the next request handler is being invoked. 


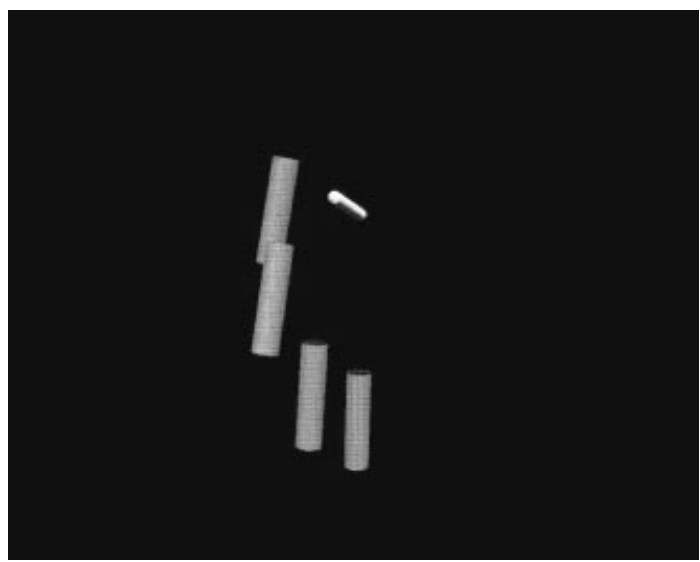

Fig. 4. The OpenGL scene as visualised on one channel of the HMD. Visible are four implants derived from the preoperative planning on the preoperative CT scan, and the drill. This scene is updated at a rate of approximately $10 \mathrm{~Hz}$.

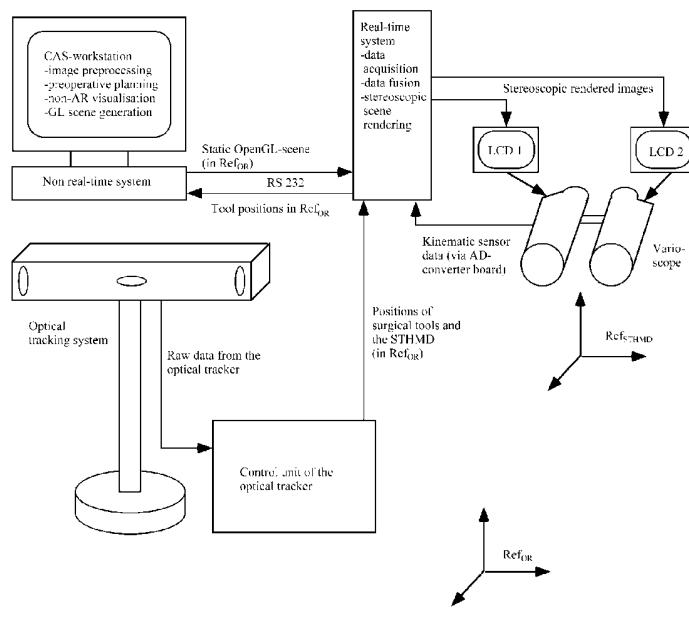

Fig. 5. The realtime control unit of the Varioscope polls data from the optical tracker at an update rate of approximately $10 \mathrm{~Hz}$. The realtime system also acquires additional data from the eight channel ADC board. Two stereoscopic scenes are rendered and displayed on two miniature VGA displays connected to two independent $\mathrm{X}$-servers. The navigation system (CAS workstation) acquires data at an update rate of approximately $1 \mathrm{~Hz}$ from the RT-system; the navigation system uses these position data to display the tool's actual position on the preoperative datasets.

\subsection{First Laboratory Assessments}

The latency in image display is a crucial problem in AR. In order to get a figure of the performance of the realtime control system of the Varioscope AR, we have analysed the single factors contributing to the overall display lag; furthermore these measurements were repeated to see whether the time lags in communication can be expected to be constant. If this is the case, predictive tracking [1] can be expected to be able to reduce the lag in display due to rapid head movements of the surgeon significantly. Measurements were taken by calling the internal timers of the Lynx OS (timer resolution: $0.01 \mathrm{~s}$ ). 
Table 2. Time requirements for data acquisition from the optical tracker and the ADC-board of the real-time workstation and the time needed for rendering the two OpenGL-scenes.

\begin{tabular}{|c||c|c|}
\hline Run No. & Data Acquisition Time $[\mathrm{s}]$ & Rendering Time $[\mathrm{s}]$ \\
\hline 1 & $0.146 \pm 0.02$ & $0.04 \pm 0.01$ \\
\hline 2 & $0.147 \pm 0.02$ & $0.04 \pm 0.01$ \\
\hline 3 & $0.147 \pm 0.02$ & $0.04 \pm 0.01$ \\
\hline 4 & $0.145 \pm 0.02$ & $0.04 \pm 0.01$ \\
\hline 5 & $0.146 \pm 0.02$ & $0.04 \pm 0.01$ \\
\hline \hline Average & $0.146 \pm 0.02$ & $0.04 \pm 0.01$ \\
\hline
\end{tabular}

\section{Results}

First of all, it turned out that common focus is easily achieved in the prototype. The HiBrite displays have turned out to be bright enough to show the OpenGL graphics in a sufficient manner without additional light sources.

The data acquisition time's repeatability was found to be in the range of the timer's resolution. Overall time requirements for accessing data from the tracker and the CAS-workstation over the serial ports was found to be $0.15 \pm 0.02 \mathrm{~s}$. The time needed for rendering the OpenGL scenes remained constant at 0.04 $\pm 0.01 \mathrm{~s}$. While evaluation of the photogrammetric registration is still under progress, first experiments at a fixed focal length of $35 \mathrm{~mm}$ resulted in a match between measured 2D display coordinates and 3D coordinates transformed back to the HMD's display of 1.7 pixel (maximum error: 3.0 pixel).

\section{Discussion}

Our current results show that a head-mounted operating microscope with the capabilities of the Varioscope AR provides a very promising approach towards introduction of AR in the operating theatre. The system can handle the problems adressed in the introduction; furthermore it can easily be connected to other navigation systems since the CAS-workstation and the realtime control unit are separated. Currently, both image update rate and latency are within reasonable limits. Since the visualisation hardware can still be improved in a cost efficient manner (by usage of OpenGL accelerated graphics boards and one or more CPUs with higher computing power) we believe that the next months will bring even increased performance. Another bottleneck is the request handler for communication with the CAS-workstation; it consumes $0.1 \mathrm{~s}$ of computing time in the realtime control system's main event loop. This is due to the use of POSIX.1 conformant serial communication between the navigation system and the realtime-unit. A faster method using Ethernet and POSIX.4 conformant timers is currently under development.

This is a paper on work-in-progress. Therefore, the most important part of evaluation, the clinical assessment, was not yet performed. Since the basic visualisation task currently is the matching of planned implant position and actual 
drill position, both static and dynamic errors in photogrammetric matching of the real world view and the computer generated view are not yet crucial. The next steps include assessment of the Kalman filter's performance for improving dynamic registration, and the accuracy of the static photogrammetric registration has to be assessed. Our current work shows, however, that our approach has the potential to bring $\mathrm{AR}$ to a wide acceptance among surgeons in a wide variety of specialties.

\section{Acknowledgment}

First of all, we wish to thank M. Lehrl, W. Pesendorfer, A. Ofner, and the staff at Life Optics for their help and cooperation. The same holds true for E. Ipp and the staff at Docter Optics Vienna. We would also like to thank F. Döcke, Planar Systems, Munich, Germany. The hardware for the Varioscope AR was to a large part made and assembled by W. Piller (L.-Boltzmann Institute of Nuclear Medicine, Vienna), S. Baumgartner, A. Taubeck, and A. Gamperl (Dept. of Biomedical Engineering and Physics, Vienna). A. Larson, D. Hanson and the staff at BIR (Mayo Clinic, Rochester/MN) were of great help during the development of VISIT. AVW, the library used for VISIT's image processing capabilities, was provided courtesy of Dr. R. A. Robb. Finally, a large number of colleagues at the clinical departments at Vienna General Hospital have provided valuable input during the development phase. This work was supported by research grant P12464-MED of the Austrian Science Foundation FWF.

\section{References}

1. R. Azuma: "Predictive Tracking for Augmented Reality", PhD thesis, UNC at Chapel Hill, (1995).

2. S. Bangay, L. Preston: "An investigation into factors influencing immersion in interactive virtual reality environments", Stud Health Technol Inform 58, 43-51, (1998).

3. W. Birkfellner, P. Solar, A. Gahleitner et al.: "Computer - Aided Implant Dentistry - An Early Report", in C. Taylor, A. Colchester (eds.): "Medical Image Computing and Computer Aided Interventions - MICCAI'99", Springer LNCS 1679, 883-891, (1999).

4. W. Birkfellner, F. Wanschitz, F. Watzinger et al.: "Accuracy of a Navigation System for Computer-Aided Oral Implantology", submitted to MICCAI 2000.

5. W. Birkfellner, P. Solar, A. Gahleitner et al.: "In-vitro assessment of a registration protocol for image guided implant dentistry", Clin Oral Impl Res, in press.

6. W. Birkfellner, K. Huber, A. Larson et al.: "A modular software system for computer-aided surgery and it's first application in oral implantology", IEEE Trans Med Imaging, in press.

7. M. Blackwell, F. Morgan, A. M. DiGioia III: "Augmented reality and its future in orthopaedics", Clin Orthop 354, 111-122, (1998).

8. T. Brinker, G. Arango, J. Kaminsky et al.: "An experimental approach to image guided skull base surgery employing a microscope-based neuronavigation system" Acta Neurochir (Wien) 140(9), 883-889, (1998). 
9. C. J. Calvano, M. E. Moran, L. P. Tackett, P. P. Reddy, K. E. Boyle, M. M. Pankratov: "New visualization techniques for in-utero surgery: amnioscopy with a three-dimensional head-mounted display and a computer-controlled endoscope", J Endourol 12(5), 407-410, (1998).

10. P. J. Edwards, A. P. King, D. J. Hawkes et al.: "Stereo augmented reality in the surgical microscope", Stud Health Technol Inform 62, 102-108, (1999).

11. P. J. Edwards, D. J. Hawkes, D. L. Hill et al.: "Augmentation of reality using an operating microscope for otolaryngology and neurosurgical guidance", J Image Guid Surg 1(3), 172-178, (1995).

12. H. Fuchs, M. A. Livingston, R. Raskar et al.: "Augmented Reality Visualization for Laparoscopic Surgery", in W. M. Wells, A. Colchester, S. Delp (eds.): "Medical Image Computing and Computer-Assisted Intervention - MICCAI'98", Springer LNCS 1496, 934 pp., (1998).

13. J. Haase: "Image-guided neurosurgery/ neuronavigation/the SurgiScope-reflexions on a theme", Minim Invasive Neurosurg 42(2), 53-59, (1999).

14. R. E. Kalman: "A New Approach to Linear Filtering and Prediction Problems", Transactions of the ASME - Journal of Basic Engineering, pp. 35 - 45, (1960).

15. M. J. Kilgard: "OpenGL Programming for the X Window System", Addison Wesley, (1996).

16. D. W. Roberts, J. W. Strohbehn, J. F. Hatch et al.: "A frameless stereotaxic integration of computerized tomographic imaging and the operating microscope", J Neurosurg 65(4), 545-549, (1986).

17. R. Tsai, "A verstaile camera calibration technique for high-accuracy $3 \mathrm{D}$ machine vision metrology using off-the-shelf TV cameras and lenses", IEEE Trans Robotics Autom, RA 3(4), 323-344, (1987).

18. A. Wagner, M. Rasse, W. Millesi et al.: "Virtual reality for orthognathic surgery: the augmented reality environment concept", J Oral Maxillofac Surg 55(5), 456462, (1997). 